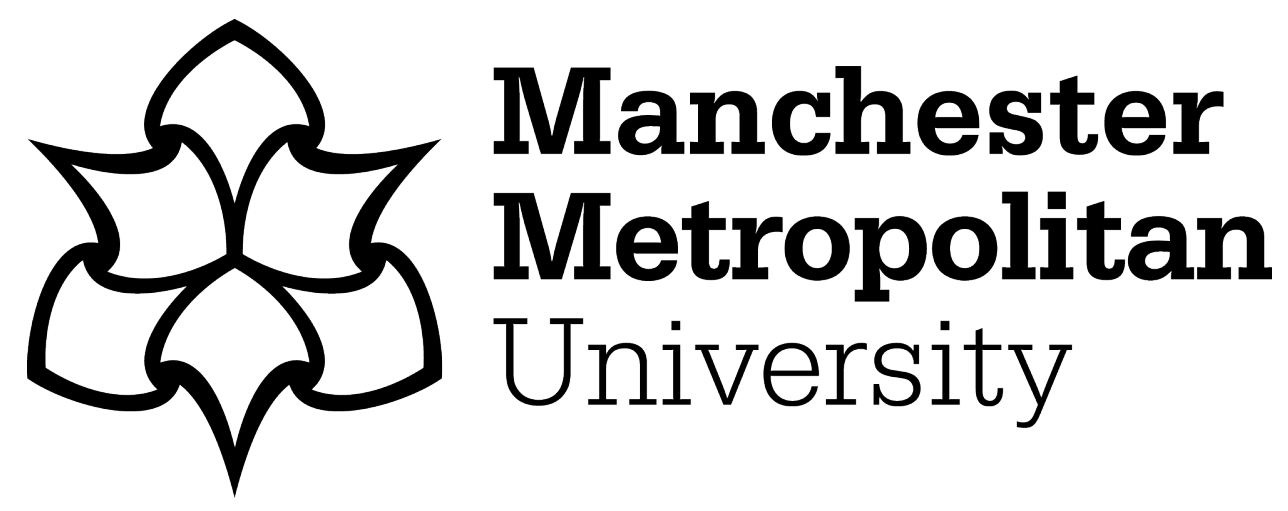

Ekpo, SC (2018) Thermal Subsystem Operational Times Analysis for Ubiquitous Small Satellites Relay in LEO. International Review of Aerospace Engineering (IREASE), 11 (2). pp. 48-57. ISSN 1973-7440

Downloaded from: https://e-space.mmu.ac.uk/620288/

Version: Published Version

Publisher: Praise Worthy Prize

DOI: https://doi.org/10.15866/irease.v11i2.13663

Usage rights: Creative Commons: Attribution-Noncommercial-No Derivative Works 4.0

Please cite the published version 


\title{
Thermal Subsystem Operational Times Analysis for Ubiquitous Small Satellites Relay in LEO
}

\author{
Sunday C. Ekpo
}

\begin{abstract}
The success of the satellite subsystems engineering depends on the optimal design, modeling, simulation, and validation of the deliverables of the conceptual and mission design objectives. This paper presents the operational times analysis of the thermal control subsystem onboard a 97-kg microsatellite in low-Earth orbit during an eclipse period. Power-storing, communication downlink and uplink, payload processing, and thermal control overpower modes were implemented for a communication mission under worst-case orbital patterns. An embedded digital temperature and lighting controller circuitry was designed and practically validated to effect a desired logic. For an average eclipse period of 34.4 mins, the operational times of the thermal subsystem at altitudes of $400 \mathrm{~km}, 500 \mathrm{~km}$, and $600 \mathrm{~km}$ are 38.6 mins, $38.1 \mathrm{mins}$, and 37.7 mins respectively. Moreover, the thermal control subsystem simulation reveals that reducing the operational times of non-thermal control subsystems during the eclipse period by $50 \%$ can result in an operational factor of safety of over 1.5. At least $10 \mathrm{~dB}$ data link transmission margin can be achieved. The reported findings show that the operational times of spacecraft subsystems overpower modes can be reconfigured in orbit to reliably sustain the operating conditions of the capability-based satellite components for ubiquitous communication. Copyright (C) 2018 The Authors.

Published by Praise Worthy Prize S.r.l. This article is open access published under the CC BY-NC-ND license (http://creativecommons.org/licenses/by-nc-nd/3.0/).
\end{abstract}

Keywords: Adaptive Device, Onboard Electronics, Operational Times, Reconfigurable, Satellite Communication, Temperature Control

\section{Nomenclature}

CFP Customer-furnished payload

$\mathrm{CO} \quad$ Communication overpower mode

CO Communication overpower

ComSat Communication satellite

$E_{\mathrm{o}} \quad$ Round-trip energy generated by the solar arrays, J

$E_{\mathrm{s}} \quad$ The energy consumptions in the power-storing

$E_{\mathrm{ss}} \quad$ Subsystem processing-overpower

$f_{\mathrm{e}} \quad$ Eclipse fraction

FPGA Field programmable gate array

HAM Highly adaptive microsatellite

HASS Highly adaptive small satellite

$H_{\text {sat }} \quad$ Altitude of the satellite, $\mathrm{km}$

JTAG Joint test action group

LCC Light controller circuit

LEO Low-earth orbit

LNA Low-noise amplifier

MetSat Meteorology satellite

OM Overpower mode

$P_{\text {i }} \quad$ The ith power mode's power consumption, W

PlaSat Planetary satellite

$P_{\max } \quad$ Maximized power mode, $\mathrm{W}$

$P_{\mathrm{n}} \quad n$ subsystem overpower, $\mathrm{W}$

PPO Payload processing overpower mode
PPO Payload processing overpower

PSM Power-storing mode

PSO Power-storing overpower

PSO Power-storing overpower mode

$P_{\mathrm{sp}} \quad$ Sunlit power generation of the solar array, W

$P_{\text {ss }} \quad$ Subsystem processing-overpower, W

$P_{\mathrm{u}} \quad$ Uplink-overpower, W

$R \quad$ The radius ratio, $R_{\mathrm{eq}} / R_{\mathrm{sat}}$

$R_{\text {eq }} \quad$ Mean equatorial radius of the Earth, $6378 \mathrm{~km}$

$R_{\text {sat }} \quad$ Geocentric radius of the satellite, $\mathrm{km}$

SE $\quad$ System engineering

SoC System-on-a-chip

STP Spacecraft team's payload

SWaP Size, weight and power

TCC Temperature controller circuit

TCO Thermal control overpower mode

TCO Thermal control overpower

$t_{\mathrm{i}}$

$t_{\max } \quad$ Maximized operational time, $\mathrm{s}$

$t_{\mathrm{n}} \quad n$ subsystem overpower time, $\mathrm{s}$

TSOT Thermal subsystem operational times

$t_{\mathrm{ss}} \quad$ Operational time of the subsystem processing

$\mathrm{OM}$

$t_{\mathrm{u}} \quad$ Uplink overpower time, $\mathrm{s}$

ULO Uplink overpower mode

ULO Uplink overpower 
$\beta \quad$ Sun-orbit-plane angle, ${ }^{\circ}$

$\mu \quad$ Gravitational constant of the Earth, $\mathrm{km}^{3} / \mathrm{s}^{2}$

$\tau_{\mathrm{o}} \quad$ Orbital period of the satellite, $\mathrm{s}$

\section{Introduction}

The range of the incident angle of the sunlight on the solar panel is a function of the inclination between the ecliptic and equatorial planes. For an inclination of $23.45^{\circ}$, the incident angle of the sunlight on the solar panel ranges from 66.55 to $90^{\circ}$. On equinox days (vernal and autumnal equinoxes), the satellite experiences the longest eclipse once a day due to the blocking of the illuminating sunlight by the Earth. When the Earth shadows a satellite, a two-fold immediate impact unfolds thus: the solar array power generation stops and the satellite temperature decreases abruptly. Thus, the ability to predict the eclipse as well as deploy reliable and fast real-time devices for the thermal and lighting control subsystems is vital for the spacecraft electrical power system design. The satellite system can experience total eclipse (umbra period lasting for 69.4 mins) or partial and total (penumbra period, lasting for $73.7 \mathrm{mins}$ ). Spacecraft systems engineers utilize the penumbra (eclipse) duration to design the electrical power subsystem. In order to meet the mission operation requirements, sustainable, reliable, reconfigurable and low-cost radiation-hardened digital circuits are vigorously being investigated and designed for spaceborne assets and integrated seamless communication networks. Every spacecraft contains sensors for measuring the amount of thermal radiation (infra-red), visible light and other radiations that arrive from the EM spectrum.

The thermal control subsystem of a space satellite maintains the right temperature margins of all onboard equipment during normal and abnormal mission operations. Passive and active modes of cooling are provided via this subsystem. It typically consists of thermostats, thermistors (temperature sensors), control electronics, multilayer blankets, louvers, fixed radiators, control electronic, coatings, tapes and heaters.

The existing system engineering analysis (SEA) margins are insufficient to sustainably address the conceptual design and mission objectives of capabilitybased small satellite missions [1] operating in the lowEarth orbit (LEO). Current space missions have heavily depended upon commercial-of-the-shelf (COTS) components and subsystems for their development to provide an economical platform for emerging technology demonstrations, scientific mission investigations, and constellations/clusters for advanced mission concepts [2].

An intelligent conceptual characterisation of the optimal operational times for the satellite subsystems is one of the critical design considerations that spacecraft engineers must qualify prior to launch [1], [3], [4]. The passive, active and adaptive components onboard a satellite operate under static and dynamic power regimes that must be estimated [4]-[6]. A balanced energy budget is established following a subsystems-level analysis of the satellite's operational times margins [7]. In-orbit performance metrics are determined by the available cost-effective and energy-efficient multiple power modes a spacecraft can accommodate [7]. In terms of a roundtrip data communication, the eclipse period presents a critical regime for the onboard electronics resources. The feasibility of fulfilling premium operational margins by small satellites is determined by their size, weight and power ( $\mathrm{SWaP}$ ) limitations. To obviate this constraint, an innovative design of embedded digitized analog RF circuits with very low power consumptions and small components footprints is required [3]. Deploying highly adaptive functional architectures would enable onboard mission data processing, and handling to be reliably performed during the eclipse duration [8]. This would lead to ubiquitous high data rate, sustainable link margin, bandwidth availability, and reliable communication subsystem.

For a given satellite mission, at least two basic power modes are feasible: a power-storing and an overpower modes. The satellite's subsystems consume more power in the overpower mode (OM) (during which the sunlit solar power generation/production for the spacecraft is less than the spacecraft's power consumption) than in the power-storing mode (PSM) (during which the illuminated solar power generation/production for the spacecraft is less than the spacecraft's power consumption). The spacecraft utilizes the PSM regime to store energy on the onboard batteries; this is utilized to sustain the mission capabilities during the OM regime. Typical overpower-mode spacecraft applications include engineering and scientific data transmission [9], onboard payload processing [10], and thermal control during eclipse periods.

\section{Small Satellite Thermal Subsystem}

In this section, the thermal subsystem engineering and the associated components are explained. The satellite system definition requirements for the thermal subsystem design, characterization, deployment and operation are presented.

\section{II.1. Thermal Subsystem Engineering}

The thermal control and management subsystem is responsible for maintaining the acceptable temperature ranges of the different components onboard a spacecraft. The radiated heat experienced by a satellite comes from the onboard electronic components, Sun and reflections from off the Earth. This radiated heat must be lost to the spacecraft environment to enable it cool down and perform optimally. Once the orbital patterns and eclipse time of a satellite are known, the radiative heat sources and sink can be estimated with an appreciable degree of accuracy.

To understand and validate the operational times of components, subsystems, and modules of a spacecraft, a 
careful investigation and analysis of the subsystems power requirements and thermal dissipation (based on the orbital patterns) must be carried out. Table I gives the core bus subsystems power allocation for the thermal subsystem of capability-based small satellite missions (including communication (ComSat); planetary (PlaSat); meteorology (MetSat); and other satellites). The two generic case studies considered are based on a customerfurnished payload (CFP) and a payload developed along with the core bus subsystems (i.e., spacecraft team's payload (STP). The thermal subsystem engineering is aimed at qualifying the different heat sources and sinks within the spacecraft environment.

TABLE I

Core Bus Subsystems POWER Allocation For Small SATEllites

\begin{tabular}{|c|c|c|c|c|c|c|c|c|}
\hline \multirow[b]{3}{*}{ Subsystem } & \multicolumn{8}{|c|}{$\begin{array}{c}\text { Percentage of Total Core Bus Subsystems Power } \\
\text { Allocation }(\%)\end{array}$} \\
\hline & \multicolumn{2}{|c|}{ ComSat } & \multicolumn{2}{|c|}{ PlaSat } & \multicolumn{2}{|c|}{ MetSat } & \multicolumn{2}{|c|}{ Other } \\
\hline & STP & CFP & STP & CFP & STP & $\mathrm{CF}$ & & $\mathrm{Cl}$ \\
\hline Thermal & 30.5 & 30.5 & 28.8 & 28.8 & 48.8 & 48.8 & 33.2 & 30.1 \\
\hline
\end{tabular}

A comprehensive system engineering analysis and design process for capability-based small satellites [1], [3] and conventional [4], [11] missions can be found in references. The next section explains a FPGA-based realtime digital thermal and lighting control subsystem design and analysis for investigating the operational times of small satellites in LEO.

\section{II.2. Real-time Thermal and Lighting Control Subsystem}

For the purpose of analyzing the system engineering implication(s) of using FPGA-based adaptive devices in satellite missions [1], [12], [13], two mission applications were considered thus:

1) A light controller circuit (LCC) for a small satellte in LEO:

- The output signal controls the electrical lighting system of the satellite (especially during the eclipse period); and

- The input signal(s) is from radiation sensors (including visible light and thermal radiation) that sense the ambient lighting condition of the satellite system.

2) A temperature controller circuit (TCC) for a small satellte in LEO:

- The input signal(s) is from a sensor (such as a thermistor) that senses the environmental condition parameter (i.e., temperature, especially during the eclipse period) onboard the satellite; and

- The output signal(s) from the digital hardware is used for enabling the controlling device (e.g., a thermostat) with recourse to the desired ambient temperature of the satellite system.

The FPGA device was utilized to design the thermal and lighting controller circuits for the small satellites missions in LEO presented in this paper. Altera's
Quartus II software and Cyclone IV E EP4CE115F29C7 FPGA device family were utilized for the implementation of the circuit. The digital component development adopted for this work involved a schematic circuit design; timing constraint and clocking analysis; functional and timing simulation; and FPGA device programming and configuration (to implement the designed digital circuit(s)).

FPGA devices support both functional and timing simulations. For $n$ number of inputs, there is a corresponding $2^{n}$ number of test vectors to be generated and used for the system simulation. The reported designed digital circuits were simulated using the Quartus II Simulator; the ModelSim application can also be utilized for the simulation of the presented FPGA designs. In a functional simulation, it is assumed that the logic blocks/elements and interconnects within the FPGA fabric are perfect with no signal propagation delays. It is mostly used to ascertain the functional integrity of a circuit at the design stage. It has a low latency since the simulation uses the logic expressions that characterise the digital circuit. The timing simulation takes the signal propagation through the circuit into consideration; it tests the fitter circuit to authenticate its functional correctness and timing. The timing analysis enables the embedded system designer to estimate the expected performance of the digital circuit with recourse to the results of the propagation delays across the several paths in the fitted actual FPGA circuit and/or chip. Following the designed circuit simulation, a physical FPGA chip is utilized to implement it. This is done by programming the configuration controls (switches) that configure the logic elements (LEs) and create the desired wiring connections. Altera's DE-series boards permits the FPGA configuration to be carried out in Joint Test Action Group (JTAG) and Active Serial (AS) modes. The JTAG mode retains its configuration information as long as the FPGA chip is powered on and loses it when it is turned off.

The AS mode uses a configuration device that employs flash memory to store the configuration data. During power-up or reconfiguration, the data is loaded onto the FPGA chip. This obviates the need to reprogram and/or reconfigure the device when the power is turnedon. This can be implemented on small satellites to sustain the optimal energy balance for a given mission.

\section{II.3. Thermal and Lighting Controller Circuit Design}

The satellite digital lighting controller circuit is shown in Fig. 1. The digital circuit can be used to provide a reliable two-way control of the spacecraft light (where logic value 0 represents an open switch); it involves the Exclusive-OR function of the two input signals. The functional and timing simulation waveforms that implement the truth table in Table II are explained in section IV.

The Altera's cyclone IV E FPGA device was used for these two simulations. 


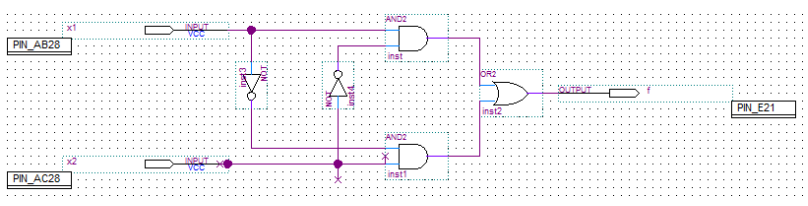

Fig. 1. A schematic design of the small satellite's LCC

TABLE II

\begin{tabular}{ccc}
\multicolumn{3}{c}{ TRUTH TABLE FOR THE DIGITAL LCC } \\
\hline \hline$x_{1}$ & $x_{2}$ & $f$ \\
\hline 0 & 0 & 0 \\
0 & 1 & 1 \\
1 & 0 & 1 \\
1 & 1 & 0 \\
\hline \hline
\end{tabular}

The digital TCC (Fig. 2) is an automatic temperature controller onboard a small satellite in LEO. It can be equally used for the spacecraft's lighting control. The heater and/or light should be "On" only when a shadow is detected (i.e., when the satellite is in the Earth's shadow (during eclipse)) or when a ground station (engineering data) command activates a switch (such as the freezing/colliding button or due to any other abnormal conditions of operation) to turn the heaters on. The heaters should only operate if they have been "Off." The logic sequence is described below:

- output: $y=1$ (Turn On Heater/Light)

- inputs:

$\circ x_{3}=1$ (Earth's Shadow Detected);

○ $x_{1}=1$ (Turn Holding the Heater and/or Light On/Open); and

○ $x_{2}=0$ (Heater Off/Shutdown)

- the Heater/Light should be turned on when:

$\circ$ the heater/light is shutdown and Earth is passing between the satellite and the Sun $\left(x_{2}=0 ; x_{3}=1\right)$;

o the Earth's shadow is detected; heater/light is Off and the switch is set to hold it open $\left(x_{3}=1 ; x_{2}=0\right.$; $x_{1}=1$ ); and

○ the temperature of the onboard electronics is below a threshold value $\left(x_{1}=1 ; x_{2}=0 ; x_{3}=0\right)$.

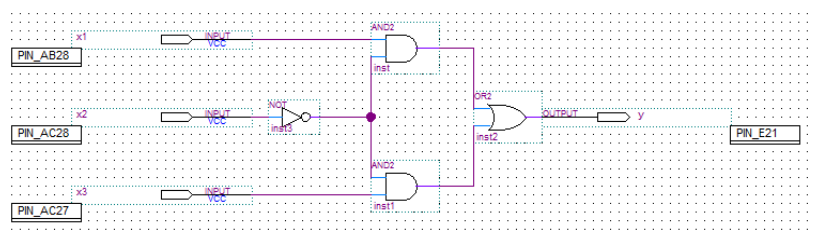

Fig. 2. A schematic design of the small satellite's TCC

TABLE III

TRUTH TABLE For The Digital TCC

\begin{tabular}{cccc}
\hline \hline$x_{1}$ & $x_{2}$ & $x_{3}$ & $y$ \\
\hline 0 & 0 & 0 & 0 \\
0 & 0 & 1 & 1 \\
0 & 1 & 0 & 0 \\
0 & 1 & 1 & 0 \\
1 & 0 & 0 & 1 \\
1 & 0 & 1 & 1 \\
1 & 1 & 0 & 0 \\
1 & 1 & 1 & 0 \\
\hline
\end{tabular}

The functional and timing simulation waveforms that implement the truth table in Table III are explained in section IV. Multiple deployments of the digital TCC can be carried out depending on the subsystem requirements definitions to satisfy the mission objectives.

\section{Thermal Subsystem Operational Times Analysis}

The operational times of the thermal subsystem of a core bus module of a small satellite is a function of the instantaneous in-orbit power modes. Each power mode is in turn affected by the prevailing orbital patterns experienced by the satellite. The thermal subsystem operational times analysis presented in this paper will consider the worst-case scenarios for the ubiquitous data relay of capability-based small satellites.

\section{III.1. Orbital Patterns Analysis}

A typical satellite system engineering considers subsystems' design variables with recourse to the orbital patterns to ensure a successful mission (and postmission) program(s). The LEO is suitable for short-term and low-cost missions up to $2000 \mathrm{~km}$. The orbital pattern parameters form the system design variables used to qualify the operational and power generation margins of spacecraft [14]. The orbital patterns are characterized by the inclination (i.e., the angular orbital sweep of a spacecraft around the Earth relative to the equator) and eccentricity (i.e., the deviation of the orbit from an Earthreferenced two-dimensional plane round-trip circle) of a spacecraft orbit.

Satellites operating non-sun-synchronous orbits experience the Earth's eclipse for a given duration during their orbital period. Hence, the eclipse period reduces the solar power generation capability of the satellite. Mathematically, the eclipse time, $t_{e}$, of a satellite is given by:

$$
t_{e}=\frac{\tau_{o}}{\pi}\left(\cos ^{-1}\left(\frac{\sqrt{1-R^{2}}}{\cos \beta}\right)\right)
$$

where:

$R=$ the radius ratio $=R_{\mathrm{cq}} / R_{\mathrm{sat}}$

$R_{\mathrm{cq}}=$ mean equatorial radius of the Earth $=6378 \mathrm{~km}$;

$R_{\text {sat }}=$ geocentric radius of the satellite, $\mathrm{km}=R_{\mathrm{cq}}+H_{\mathrm{sat}}$;

$H_{\text {sat }}=$ altitude of the satellite, $\mathrm{km}$;

$\beta=$ Sun-orbit-plane angle, ${ }^{\circ}$;

$\tau_{0}=$ orbital period of the satellite, $\mathrm{s}$;

$\mu=$ gravitational constant of the Earth $=398,600.4418$ $\mathrm{km}^{3} / \mathrm{s}^{2}$.

The eclipse fraction, $f_{\mathrm{e}}$, is obtained from (1) as follows:

$$
f_{e}=\frac{1}{\pi}\left(\cos ^{-1}\left(\frac{\sqrt{H_{s a t}^{2}+2 R_{e q} H_{s a t}}}{\left(R_{e q}+H_{s a t}\right) \cos \beta}\right)\right)
$$

The orbital period of the satellite, $\tau_{\mathrm{o}}$, is given by: 


$$
\tau_{o}=2 \pi \sqrt{R_{\text {sat }}^{3} / \mu}
$$

The energy generation of the solar panels is $0 \mathrm{~W}$ during the eclipse period, and the satellite maintains the system functionalities using the onboard storage batteries. The worst-case sun-orbit plane angle, $\beta$, occurs at $0^{\circ}$ when the satellite experiences the maximum Earth's eclipse; the best-case $\beta$ occurs at $\pm 90^{\circ}$ when the orbit never enters the Earth's eclipse.

\section{III.2. Operational Modes of a Small Satellite}

For a $N$-power mode system involving several power modes for single and multiple missions and post-mission applications, the following power consumptions are feasible:

1) Power-storing;

2) Communication (downlink and uplink)-overpower, $P_{d u}$, with a corresponding time, $t_{d u}$;

3) Uplink-overpower, $P_{u}$, with a corresponding time, $t_{u}$;

4) Subsystem processing-overpower, $P_{s s}$, with a corresponding time, $t_{s s}$; and

5) Other overpower modes, $P_{n}$, with a corresponding time, $t_{n}$, where $n=1,2, \ldots$.

The prevailing modes of the various subsystems of a small satellite can be accommodated and adapted to fulfil the conceptual design and mission objectives.

\section{III.3. Thermal Subsystem Operational Times Analysis}

The eclipse period is a fraction of a satellite's orbital period and affects the total energy reserve of the satellite. The round-trip total energy generated by the satellite's solar arrays, $E_{\mathrm{o}}$, is given by:

$$
E_{o}=P_{s p}\left(\tau_{o}-t_{e}\right)
$$

where $P_{\mathrm{sp}}$ is the average sunlit power generation (in Watts) of the solar array in a given orbit.

The power consumptions for a two-power mode system can be modelled by considering the following:

1) Power-storing, $P_{\mathrm{s}}$; and

2) Subsystem processing-overpower, $P_{\mathrm{ss}}$.

From (4), the total generated energy of the satellite during a round-trip for a two-power mode system can be obtained thus:

$$
E_{o}=P_{s} t_{s}+P_{s s} t_{s s}
$$

where

$t_{\mathrm{s}}=$ the operational times of the power-storing in second; $t_{\mathrm{ss}}=$ subsystem overpower modes in second.

Equation (5) represents the energy consumptions in the power-storing, $E_{\mathrm{s}}=P_{\mathrm{s}} t_{\mathrm{s}}$, and subsystem processingoverpower, $E_{\mathrm{ss}}=P_{\mathrm{ss}} t_{\mathrm{ss}}$, modes. The operational time of the subsystem processing-overpower mode, $t_{\mathrm{ss}}$, is a function of the orbital period and eclipse time and obtained as:

$$
t_{s s}=\tau_{o}-\left(t_{s}+t_{e}\right)
$$

For a two-power mode system, the operational time of the subsystem processing-overpower mode is derived from (4) to (6) as follows:

$$
t_{s s}=\frac{E_{o}-P_{s}\left(\tau_{o}-t_{e}\right)}{P_{s s}-P_{s}}
$$

The in-orbit mission's energy budget balance is obtained as a sum of the energy requirements of the satellite's subsystems and given by:

$$
E_{o}=E_{s}+E_{d u}+E_{u}+E_{s s}+E_{1}+E_{2}+\ldots+E_{N}
$$

The overall operational time of the subsystem processing-overpower mode is a function of the powerstoring time, eclipse time, downlink time, uplink time and other prevailing times of $N$-power modes. This can be obtained mathematically as:

$$
t_{s s}=\tau_{o}-\left(t_{s}+t_{e}+t_{d u}+t_{u}+t_{1}+t_{2}+\ldots+t_{N}\right)
$$

From (8) and (9), the overall in-orbit operational time of the subsystem processing-overpower mode, $t_{\mathrm{ss}}$, is derived as:

$$
\begin{aligned}
t_{s s}= & \frac{E_{o}-P_{s}\left(\tau_{o}-\left(t_{e}+t_{d u}+t_{u}+t_{1}+t_{2}+\ldots+t_{N}\right)\right)}{P_{s s}-P_{s}}+ \\
& \frac{-\left(P_{1} t_{1}+P_{2} t_{2}+\ldots+P_{N} t_{N}\right)}{P_{s s}-P_{s}}
\end{aligned}
$$

Equation (10) can be re-written thus:

$$
t_{s s}=\frac{E_{o}+P_{s}\left(\sum_{i=1}^{N-2} t_{i}+t_{e}-\tau_{o}\right)-\left(\sum_{i=1}^{N-2} P_{i} t_{i}\right)}{P_{s s}-P_{s}}
$$

where $P_{\mathrm{i}}$ and $t_{\mathrm{i}}$ represent the $i$ th power mode's power consumption and operational time respectively. The summations in (8) are implemented respecting all the overpower modes except the power-storing and the particular subsystem processing-overpower mode. A comprehensive temporal enumeration of the power consumptions and corresponding operational times of the power modes of interest must be estimated in order to obtain the $t_{\mathrm{ss}}$ for the satellite mission. The correct estimates of the power consumptions of the powerstoring mode and the power mode whose operational time are required to compute the $t_{\mathrm{ss}}$.

In spacecraft missions, it is vital to maximize the operational time of a power mode. Hence, (11) is adjusted to obtain the maximized power mode, $P_{\max }$. The corresponding maximized operational time, $t_{\max }$, is given by: 


$$
t_{\max }=\frac{E_{o}+P_{s}\left(\sum_{i=1}^{N-2} t_{i}+t_{e}-\tau_{o}\right)-\left(\sum_{i=1}^{N-2} P_{i} t_{i}\right)}{P_{\max }-P_{s}}
$$

Equation (12) enables the modeling of the operational times of the subsystems and modules of a spacecraft based on the systems definition requirements, conceptual objectives, and mission design variables.

\section{TSOT Simulation Parameters}

To simulate the behavior of the digital circuit, a sufficient number of input valuations would be applied to observe the expected output values. For a large circuit, a relatively small and representative values of the huge input valuations are chosen. For the designed small satellite's LCC, the desired functional and timing simulations were set to run from 0 to $200 \mathrm{~ns}$. The four test vectors were applied using four 50-ns time intervals. The logic states timings were set as follows: $x_{1}$ [0: 0 to $100 \mathrm{~ns} ; 1: 100$ to $200 \mathrm{~ns}$ ]; $x_{2}$ [1:50 to $100 \mathrm{~ns} ; 1: 150$ to 200 ns].

A similar procedure was followed for the FPGA-based digital TCC for a small satellite mission. The TCC is shown in Fig. 2. For the designed small satellite's TCC, the desired functional and timing simulations were set to run from 0 to $400 \mathrm{~ns}$. The eight test vectors were applied using four 50-ns time intervals. The logic states timings were set as follows: $x_{1}$ [0: 0 to $200 \mathrm{~ns} ; 1: 200$ to $400 \mathrm{~ns}$ ]; $x_{2}$ [1: 100 to $200 \mathrm{~ns} ; 1: 300$ to $400 \mathrm{~ns}$ ]; $x_{3}$ [1: 50 to 100 ns; 1: 150 to $200 \mathrm{~ns} ; 1: 250$ to $300 \mathrm{~ns} ; 1: 350$ to $400 \mathrm{~ns}$ ]. The operational times of the thermal control processing overpower, payload processing overpower and communication-overpower modes for a candidate communication mission are obtained with recourse to the orbital pattern parameters (Table IV).

Table $\mathrm{V}$ presents the orbital patterns for the TSOT analysis of the case study communication mission in LEO.

From the system engineering analysis of highly adaptive microsatellites (HAM) in LEO [14] and (13) and (14), an altitude of $500 \mathrm{~km}$ is chosen for the case study communication mission using a microsatellite with an on-orbit dry mass of $97 \mathrm{~kg}$. For a Class 2 design (i.e., next-generation spacecraft), a contingency factor of $25 \%$ is chosen for the microsatellite mass and power margins. Assuming that four solar panels are deployed by the microsatellite in orbit, the maximum generated electrical power is $116.83 \mathrm{~W}$ [1]. The calculated corresponding core bus power is $59.27 \mathrm{~W}$. Hence, the power allocation for the thermal subsystem is $18.08 \mathrm{~W}$. During the eclipse period, the thermal subsystem would need more than this power value to maintain the right operating temperature range for the different electronic components onboard the microsatellite. The prevailing orbital pattern limits how much power can be generated and hence, the thermal control subsystem must enter the "overpower" operational mode.
TABLE IV

DESIGN PARAMETERS FOR SATELLITE TSOT MODELLING

\begin{tabular}{|c|c|c|}
\hline Parameter & Description & Value \\
\hline Spacecraft mass (kg) & Microsatellite & $95 \leq M \leq 100$ \\
\hline \multirow[t]{2}{*}{ TSOT (mins) } & $\begin{array}{l}\text { Payload processing } \\
\text { Communication }\end{array}$ & $\begin{array}{c}t_{\mathrm{p}}>0 \\
t_{\mathrm{DL}}>0 ; t_{\mathrm{UL}}>0\end{array}$ \\
\hline & Thermal control & $t_{\mathrm{tc}}>0$ \\
\hline $\begin{array}{l}\text { Power-storing power, } \\
P_{\mathrm{s}}(\mathrm{W})\end{array}$ & $\begin{array}{l}\text { Power is generated; no } \\
\text { payload processing and } \\
\text { communication }\end{array}$ & $P_{\mathrm{s}}>0$ \\
\hline $\begin{array}{l}\text { Max. eclipse time, } \\
t_{\mathrm{e}}(\mathrm{mins})\end{array}$ & $\begin{array}{l}\text { Payload processing; } \\
\text { communication and } \\
\text { thermal control; no } \\
\text { power is generated. }\end{array}$ & $t_{\mathrm{e}} \geq 35.0$ \\
\hline$\beta$ range $\left(^{\circ}\right)$ & Sun-orbit-plane angle & 0 \\
\hline $\begin{array}{l}\text { Orbital period, } \\
\tau_{\mathrm{o}}(\text { mins })\end{array}$ & $\begin{array}{l}\text { Power is generated; } \\
\text { Payload processing and } \\
\text { Communication. }\end{array}$ & $90 \leq \tau_{\mathrm{o}} \leq 100$ \\
\hline Inclination $\left({ }^{\circ}\right)$ & Closed orbit; Elliptical & $22.5 \leq I_{\text {sat }} \leq 157.5$ \\
\hline $\operatorname{RAAN}\left({ }^{\circ}\right)$ & $\begin{array}{l}\text { Right ascension of the } \\
\text { ascending node. }\end{array}$ & $0 \leq R A A N \leq 90$ \\
\hline Altitude $(\mathrm{km})$ & LEO & $400 \leq H_{\text {sat }} \leq 2500$ \\
\hline
\end{tabular}

TABLE V

ORBITAL PATTERNS FOR THE COMSAT MISSION IN LEO

\begin{tabular}{cccc}
\hline \hline Orbit & Altitude, $H_{\text {sat }}(\mathrm{km})$ & Inclination $\left(^{\circ}\right)$ & RAAN $\left(^{\circ}\right)$ \\
\hline Inclination & 400 & 22.5 & 30 \\
Inclination & 500 & 45 & 60 \\
Inclination & 600 & 67.5 & 90 \\
Inclination & 400 & 112.5 & 30 \\
Inclination & 500 & 135 & 0 \\
Inclination & 600 & 157.5 & 0 \\
\hline \hline
\end{tabular}

Table VI contains the power-storing overpower (PSO), communication overpower (CO), uplink overpower (ULO), payload processing overpower (PPO) and thermal control overpower (TCO) modes for the case study microsatellite. For the purpose of modelling the impact of implementing five power modes during the eclipse period, an operational time of 10 minutes is assumed for the communication overpower, uplink overpower and payload processing overpower modes.

TABLE VI

ENERGY RESERVE BUDGET FOR THE COMSAT MISSION IN LEO

\begin{tabular}{lccccc}
\hline \hline \multirow{2}{*}{ Subsystem } & \multicolumn{5}{c}{ Power Mode's Power Consumption (W) } \\
\cline { 2 - 6 } & PSO & CO & ULO & TCO & PPO \\
\hline ADC & 2 & 5 & 5 & 5 & 5 \\
C\&DH & 3 & 15 & 15 & 15 & 10 \\
Uplink & 3 & 23 & 20 & 15 & 15 \\
Downlink & 5 & 30 & 30 & 15 & 24 \\
Payload & 0 & 23 & 23 & 15 & 40 \\
Board & 1 & 2 & 2 & 5 & 5 \\
Thermal & 0 & 10 & 10 & 40 & 10 \\
EPS & 5 & 10 & 10 & 15 & 10 \\
Propulsion & 1 & 0 & 5 & 5 & 5 \\
Mechanics & 0 & 0 & 0 & 0 & 0 \\
\hline Total & 20 & 118 & 120 & 130 & 124 \\
\hline \hline
\end{tabular}

\section{Results and Discussion}

The functional (Fig. 3) and timing (Fig. 4) simulations are similar with no noticeable delay in producing a change in the signal $f$ from the time when the input signals, $x_{1}$ and $x_{2}$, change their values. For a cyclone II device, there is a delay of about $6 \mathrm{~ns}$ in producing a change in the signal $f$ from the time when the input signals, $x_{1}$ and $x_{2}$, change their values. This delay is due 
to the propagation delays in the logic element and the wires in the FPGA device.

The designed digital LCC was implemented on an actual FPGA chip (Fig. 5) to realize the truth table shown in Table II. The JTAG mode was utilized for the programming/configuration setting. The hardware setup for downloading the configuration data involved adding a USB Blaster (USB-0) interface for the data link between the PC and the FPGA board. The FPGA-based digital circuit was tested by changing the switch positions on the DE2-115 prototyping board (with recourse to the four test vectors) to predict its performance during an actual spacecraft mission.

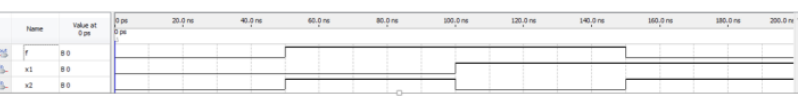

Fig. 3. Functional Simulation Result of the Digital LCC

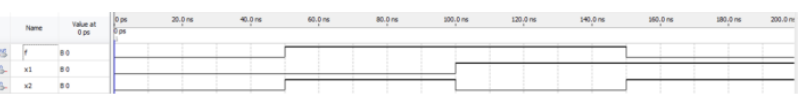

Fig. 4. Timing Simulation Result of the Digital LCC

Both the functional (Fig. 6) and the timing (Fig. 7) simulations confirm the correctness of the digital circuit aimed at implementing the desired control performance while onboard a small satellite in LEO. The output at each temporal resolution is a function of the logic states combination of the three inputs.

The designed digital TCC was implemented on an actual FPGA chip (Fig. 8) to realize the truth table shown in Table III.

Fig. 9 shows the measured power consumption profiles of the Cyclone IV E FPGA device during the TCC implementation. The pre-configuration power of the FPGA board increased abruptly from approximately 1.2 $\mathrm{W}$ to approximately $3.7 \mathrm{~W}$ in 8 secs when the board was turned on. It then decreased to about $3.5 \mathrm{~W}$ before settling at $3.6 \mathrm{~W}$ during the programming and configuration of the FPGA device.

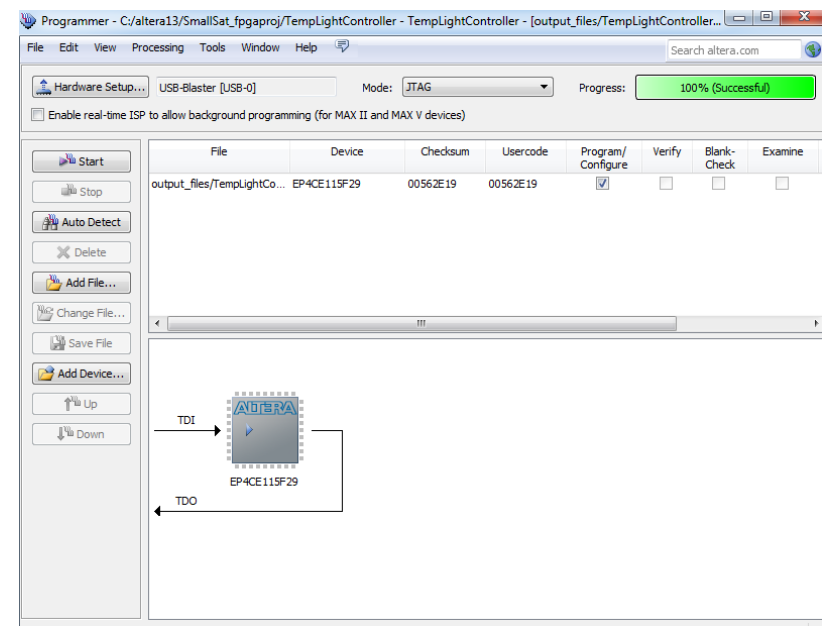

Fig. 5. Programming and configuring the FPGA device for the digital LCC

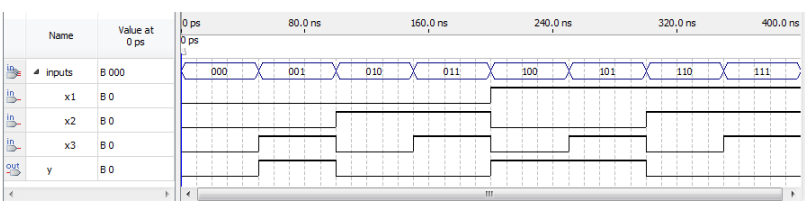

Fig. 6. Functional simulation waveform of the digital TCC

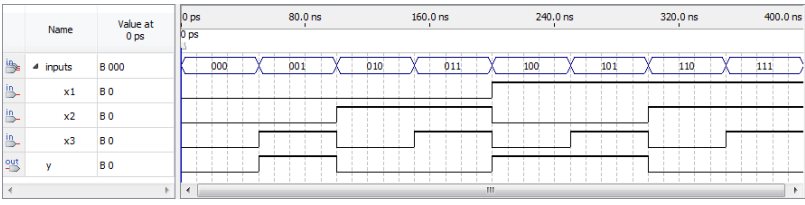

Fig. 7. Timing simulation waveform of the digital TCC

As in most space systems designs, the temperature onboard a spacecraft might vary at different locations within the system due to directional and/or selective impacts of space radiations. This can cause the various subsystems onboard a small satellite to be exposed to different temperature profiles at different times of the day and season. This kind of scenario would benefit from a real-time, high-speed reconfigurable device such as a differential digital TCC feeder.

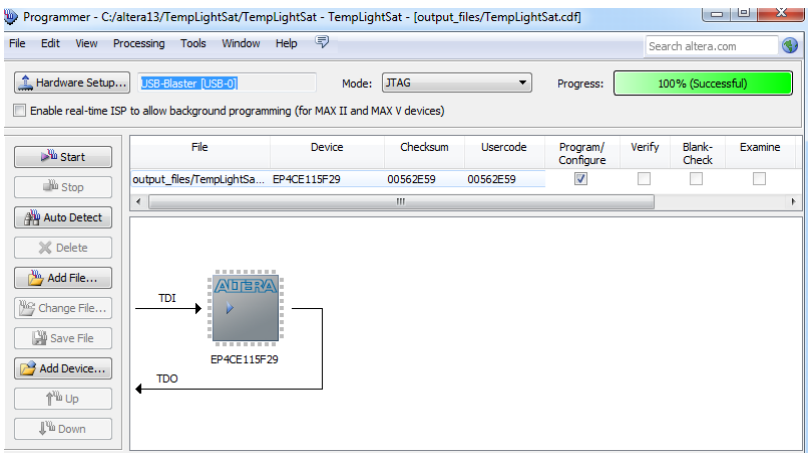

Fig. 8. Programming and configuring the FPGA device for the digital TCC

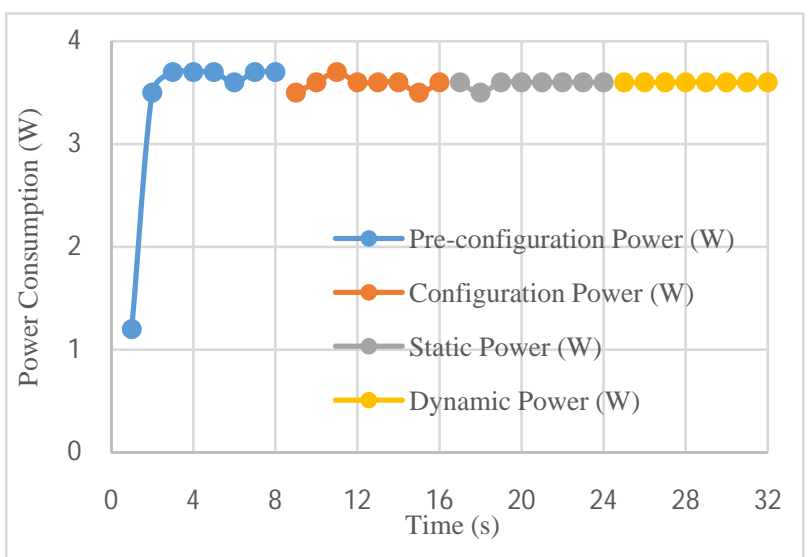

Fig. 9. Measured power consumption of the FPGA device for the digital TCC

An important information of great interest to a digital hardware designer is the speed at which a given circuit implements its logic functions. The maximum frequency, $f_{\text {max }}$, for clocking the digital circuit is a good measure of 
the speed of implementation. $f_{\max }$ depends on the longest delay experienced along any path between two concurrently-clocked registers. A timing analysis to determine the performance of the differential TCC was carried out using the Quartus ${ }^{\circledR}$ II software. The $f_{\max }$ summary for multiple models of analysis under different operating conditions were done. The Slow $1100 \mathrm{mV} 85 \mathrm{C}$ Model shows that the maximum frequency for the differential TCC implemented on the Cyclone IV E FPGA chip is $274.05 \mathrm{MHz}$. Timing constraints were created and analyzed for the differential TCC to operate at a clock frequency of $300 \mathrm{MHz}$. This was carried out using Altera's Quartus ${ }^{\circledR}$ II TimeQuest Timing Analyzer tool. The analysis yielded a maximum clock frequency of $275.56 \mathrm{MHz}$.

With the advances in adaptive devices technologies, embedded FPGAs can be implemented to address the SWaP limitations of capability-based small satellites. An embedded FPGA is an IP block that allows a complete FPGA to be incorporated in a system-on-a-chip (SoC) module or any kind of integrated electronic circuit (including digitized analog RF). Embedded FPGAs support customization. Hence, a small footprint of reprogrammable logic on the peripheral bus can be implemented to program the serial interface register transfer language (RTL). This would enable any serialinterface protocol to be deployed on-demand. Moreover, an embedded FPGA enables in-system reprogrammability.

It allows a chip, and the electronic system it resides in, to be reprogrammed to handle new/emergent communication standards that did not exist when the chip was designed. Battery life in short-term space mission applications is critical due to the SWaP limitations of capability-based small satellites. Most battery usage is attributed to the frequent, repetitive, low-level tasks, rather than the infrequent more complex operations. Several low-level digital signal processing functions could be processed by a small block of embedded FPGAs as fast or faster than a competing microprocessor, but using $2 \times$ to $5 \times$ less energy.

The embedded FPGA offloads the repetitive, lowlevel tasks at lower energy and the microprocessor is only engaged when it is time for a more complex task. This helps to extend the battery life onboard the small satellite for successful mission applications and possible post-mission reuse.

Table VII reports the orbital period, maximum eclipse time, and the operational times of the thermal control subsystem with recourse to the orbital patterns in Table $\mathrm{V}$ and the modelling parameters.

TABLE VII

TSOT ANALYSIS FOR THE COMSAT MISSION IN LEO

\begin{tabular}{cccc}
\hline \hline $\begin{array}{c}\text { Altitude, } \\
H_{\text {sat }}(\mathrm{km})\end{array}$ & $\begin{array}{c}\text { Orbital } \\
\text { Period, } \tau_{\mathrm{o}} \\
(\text { min. })\end{array}$ & $\begin{array}{c}\text { Max. eclipse } \\
\text { time, } t_{\mathrm{emax}} \\
(\mathrm{min} .)\end{array}$ & $\begin{array}{c}\text { TSOT, } t_{\mathrm{tc}} \\
(\mathrm{min} .)\end{array}$ \\
\hline 400 & 92.6 & 36.1 & 24.8 \\
500 & 94.6 & 35.7 & 24.4 \\
600 & 96.7 & 35.5 & 24.0 \\
\hline \hline
\end{tabular}

It is obvious from Table VII that the corresponding operational time of the thermal subsystem decreases as the altitude of deployment increases. The same pattern is also observed with the orbital period and the eclipse time of the satellite.

Though the maximum eclipse time for the case study mission is 35.7 mins, the operational time of the thermal control during the eclipse period is approximately 24.4 mins when the operational times of the communication, uplink and payload processing overpower modes are set to 10 mins. This result presents two challenges. Firstly, the thermal control and management of the satellite can be constrained to cover the eclipse period through power rationing.

Alternatively, the operational times of the communication, uplink and payload processing overpower modes can be reduced to allow for more power to be reserved for the optimal control of the temperature range of the different components onboard the microsatellite during the eclipse period. If the second option is implemented and the operational times of the other three power modes are $50 \%$ less, the operational times of the thermal subsystem at $400 \mathrm{~km}, 500 \mathrm{~km}$, and $600 \mathrm{~km}$ are $38.6 \mathrm{~min}, 38.1 \mathrm{mins}$, and $37.7 \mathrm{mins}$ respectively. This implementation shows that the thermal control subsystem can reliably sustain the operating conditions of the satellite components over the eclipse period by a factor of safety of approximately 1.6.

A further novelty of the presented spacecraft subsystems operational times analysis is in using the technique to optimize communication link margins. For a capability-based spacecraft with different multiple transponder subsystems, achieving a good signal-to-noise ratio (SNR) for LEO mission applications is challenging. With the developed and reported operational times modeling technique, multi-band, multi-standards radio communication channel links performance metrics can be enhanced.

Fig. 10 shows the SNRs for a radio frequency communication receiver operating at $\mathrm{C}-(4-8 \mathrm{GHz}), \mathrm{X}$ $(8-12 \mathrm{GHz})$ and $\mathrm{K}-(18-21.6 \mathrm{GHz})$ bands with sensitivities of $-96.51 \mathrm{dBm},-94.51 \mathrm{dBm}$ and $-96.01 \mathrm{dBm}$ respectively. Each low-noise amplifier (LNA) can be configured in-orbit for a real-time round-trip data reception and processing over the same communication range.

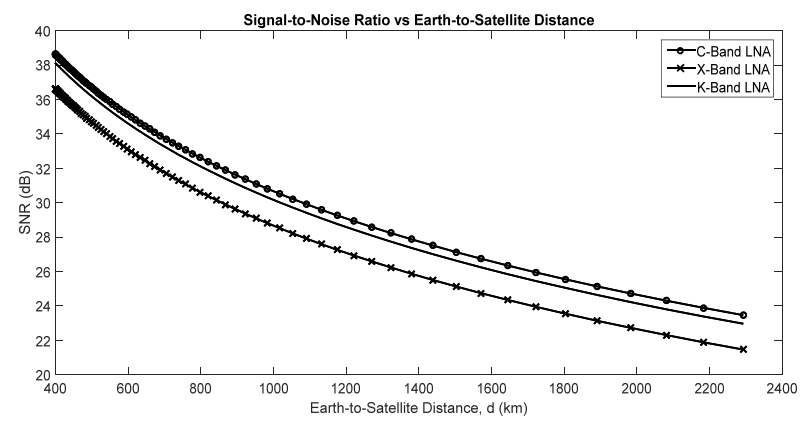

Fig. 10. Signal-to-noise ratios of a satellite receiver subsystem at C-, Xand K-bands in LEO 
It is evident from Fig. 10 that the $\mathrm{C}$ - and K-bands LNAs outperform the X-band LNA due to their achieving lower minimum detectable signals at those bands by adjusting their operational times constraints. Thermal noise increases the noise figure of LNAs. Hence, providing the right temperature control for onboard electronics enhances the operational margins required for a given mission application. Furthermore, space-borne resource allocation within a distributed satellite cluster network [15], [16] can be improved through implementing optimal subsystems operational times regimes.

Multi-beamforming that operates with the conventional architecture with a limited noise floor level has been proposed for distributed satellite relay clusters [15]. With advanced residual noise extraction techniques employed within an adaptive broadband beamformer [18], subsystems operational times can be optimized for a high throughput and seamless inter-satellite beamforming links for distributed satellite networks. As these have not been carried out and/or reported in the literature, this work promises to enable future spacecraft systems perform real-time in-orbit subsystem-level adjustments to deliver optimal operational margins.

\section{Conclusion}

This paper presents a thermal subsystem operational times for ubiquitous small satellites relay. Five power modes were analyzed and utilized to develop an operational time model for the thermal control subsystem of a microsatellite under different orbital patterns. The findings indicate that the operational times vary for different orbital and eclipse periods. A case study communication mission at an altitude of $500 \mathrm{~km}$ was considered.

For an operational time of 10 minutes for each of the communication overpower, uplink overpower and payload overpower modes, the operational time of the thermal control during the eclipse period is approximately 24.4 mins. The research reveals that the lower the operational times of non-critical overpower modes, the higher the operational factor of safety for the mission during the eclipse period. Furthermore, embedded field programmable gate array devices can be utilized as an IP block to achieve in-orbit in-system reprogrammability, customization and extended onboard battery life for seamless airborne communication application. High data rate communication links can be configured on-demand for critical data transmissions in real-time. Thus, selective subsystems operations can be implemented onboard the spacecraft for cost-effective and optimal data relay margins.

This paper utilized measured parametric data of capability-based small satellite subsystems to derive the power estimating relationships and operational times models. Hence, they are limited to adaptive small satellite system engineering design. However, the presented design parameters have considered enabling and emerging technologies and so can be extended and scaled-up to address the mission definitions requirements of medium and large spacecraft.

\section{Acknowledgements}

The author wishes to thank the Akwa Ibom State Government of Nigeria for sponsoring this research at The University of Manchester, UK, and the Engineering \& Materials Science Research Centre, Manchester Metropolitan University, UK for sponsoring extra research equipment through its open bid scheme.

\section{References}

[1] S. Ekpo, D. George, A System Engineering analysis of highly adaptive small Satellites, IEEE Systems Journal, 7 (2013), 642648.

[2] L. B. Rainey, Space Modeling and Simulation: Roles and Applications Throughout the System Life Cycle. (California: The Aerospace Press, 2004).

[3] S. Ekpo, D. George, A Deterministic Multifunctional Architecture Design for Highly Adaptive Small Satellites, International Journal of Satellite Communication Policy and Management, 1 (2012), 174-194.

[4] C. Brown, Elements of Spacecraft Design. (Reston, VA: AIAA, 2002, pp. 1-43).

[5] S. Ekpo, D. George, A System-based Design Methodology and Architecture for Highly Adaptive Small Satellites, The 4th Annual IEEE Int. Systems Conference, 516-519 (2010).

[6] H. Helvajian, S. W. Janson, Small Satellites: Past, Present, and Future. Reston (VA: AIAA, 2008, pp. 559-569, 773).

[7] S. Ekpo, D. George, B. Adebisi, A Multicriteria Optimisation Design of SPSE for Adaptive LEO Satellites Missions Using the PSI Method, The AIAA Space Conference \& Exposition, 1-19 (2013).

[8] R. Hassan, W. Crossley, Spacecraft reliability-based design optimization under uncertainty including discrete variables, $J$. Spacecraft Rockets, 45 (2008), 394-405.

[9] S. Ekpo, D. George, Impact of Noise Figure on a Satellite Link Performance, IEEE Communications Letters, 15 (2011), 977-979.

[10] S. Ekpo, D. George, 4-8 GHz LNA design for an adaptive small Satellite Transponder using InGaAs PHEMT Technology, The 11th IEEE Wireless \& Microwave Conference Proc., 1-4 (2010).

[11] W. J. Larson, J. R. Wertz, Space Mission Analysis and Design, second ed. (Torrance, CA: Microcosm, Inc. and Kluwer Academic Publishers, 1992).

[12] H. Schmitz, Application Examples: How to Use FPGAs in Satellite Systems, Actel Corporation Publication, California, 1-7 (2010).

[13] H. Helvajian, S. W. Janson, Small Satellites: Past, Present, and Future. (AIAA, Inc., Virginia, 2008, pp. 771-810).

[14] S. C. Ekpo, et al., A system-level multicriteria modelling of payload operational times for communication satellite missions in LEO, Recent Progress in Space Technology, 4 (2014), 67-77.

[15] X. Zhong et al., Joint Downlink Power and Time-Slot Allocation for Distributed Satellite Cluster Network Based on Pareto Optimization, IEEE Access Journal, 5 (2017), 25081-25094.

[16] F. H. Dong, et al., Topology structure design and performance analysis on distributed satellite cluster networks, Proc. 4th ICCSNT, 881-884, (2015).

[17] Q.Y. Yu et al., Virtual multi-beamforming for distributed satellite clusters in space information networks, IEEE Wireless Commun., 23 (2016) 95-101.

[18] S. Ekpo, B. Adebisi,A. Wells, Regulated-element Frost Beamformer for Vehicular Multimedia Sound Enhancement and Noise Reduction Applications, IEEE Access Journal, 5 (2017) 27254-27262. 


\section{Authors' information}

Dept. of Electrical \& Electronic Engineering,

School of Engineering, Manchester Metropolitan University,

Manchester, M1 5GD, United Kingdom.

E-mails: $\quad$ scekpo@ieee.org

S.Ekpo@mmu.ac.uk

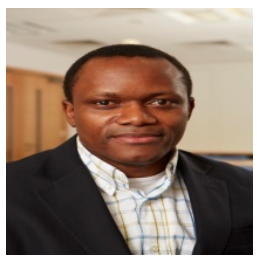

Sunday C. Ekpo obtained the M.Sc. degree in communication engineering from the University of Manchester, Manchester, U.K. in 2008 and completed his Ph.D. degree in electrical and electronic Engineering at the same institution in 2011. He is a lecturer in electrical and electronic engineering at the Manchester Metropolitan University, UK.

He has published over 40 peer-reviewed and refereed technical papers in communication and space systems engineering. His specialty spans adaptive satellite system design; multiphysics characterization of RF and optical transceivers; multi-objective system engineering analysis; intelligent sensors design; and internet of things implementations.

Dr Ekpo is a member of the IET (UK), the AIAA (USA), and the Applied Computational Electromagnetics Society (USA) and a Fellow of the Higher Education Academy (UK). 